CASE REPORT

\title{
Klippel-Trenaunay syndrome with small vessel pulmonary arterial hypertension
}

\author{
S Ulrich, M Fischler, B Walder, T Pfammatter, R Speich
}

Thorax 2005;60:971-973. doi: 10.1136/thx.2004.023184

A patient with Klippel-Trenaunay syndrome and pulmonary arterial hypertension not associated with chronic thromboembolic pulmonary hypertension is described. It is hypothesised that pulmonary arterial hypertension is another complication associated with the Klippel-Trenaunay syndrome, possibly due to haemodynamic changes of small vessel abnormalities.

$\mathrm{T}$ he Klippel-Trenaunay syndrome (KTS) is a rare congenital disorder characterised by vascular malformations including varicose veins, cutaneous capillary malformations of the port wine type, and hypertrophy of the bones and/or soft tissue. ${ }^{12}$ In contrast to a syndrome described a few years later by Parkes and Weber (Parkes-Weber syndrome $)^{3}$ with a worse prognosis related to early hyperdynamic circulatory heart failure and/or significant peripheral ischaemia, arteriovenous communications in KTS-if found at all-are only minor and without significant flow. ${ }^{4} \mathrm{~A}$ propensity of KTS to recurrent thromboembolic events is well known with a reported incidence of up to $22 \%^{1}$ and, in a subset of patients, this may lead to chronic thromboembolic pulmonary hypertension (CTEPH). ${ }^{56}$ We report a patient with KTS and pulmonary arterial hypertension (PAH) not associated with CTEPH, and hypothesise that PAH is another complication associated with KTS possibly due to haemodynamic changes of small vessel abnormalities.

\section{CASE REPORT}

A 45 year old man with left sided truncal KTS developed progressive exertional dyspnoea and fatigue over the previous 2 years. His past medical history was noteworthy for several abdominal and vascular operations due to varices and bleeding related to KTS, but splenectomy had never been performed. He had not suffered from joint pain or other symptoms suggesting connective tissue disease, nor from fatigue or a tendency to daytime sleepiness suggesting obstructive sleep apnoea. Any use of anorexigens or stimulants was neglected. Smoking history revealed 30 pack years, ceased 20 years earlier. During evaluation for exertional dyspnoea, pulmonary hypertension $(\mathrm{PH})$ was strongly suspected by echocardiography due to pathological septal movement. The pulmonary artery systolic pressure could not be estimated. Right heart catheterisation confirmed a raised mean pulmonary arterial pressure (PAP) of $47 \mathrm{~mm} \mathrm{Hg}$ with a normal pulmonary arterial occlusion pressure of $12 \mathrm{~mm} \mathrm{Hg}$. The patient declined any treatment.

Eleven months later he had to be admitted urgently with right heart failure and newly diagnosed atrial flutter. After medical stabilisation and correction of the arrhythmia, clinical signs of right heart failure were no longer present. Body weight returned to his usual $77 \mathrm{~kg}$ (body mass index $25.1 \mathrm{~kg} / \mathrm{m}^{2}$ ), the second heart sound remained prominent, no cardiac murmurs or pulmonary rales were found, the liver and spleen were not enlarged, and there was no jugular vein distension. The left side of the body was slightly hypertrophic and a large port vine type cutaneous hyperpigmentation containing vascular ectasias was present. Laboratory tests revealed polycythaemia (haemoglobin $18.4 \mathrm{~g} / \mathrm{dl}$ ), hyperuricaemia $(679 \mu \mathrm{mol} / \mathrm{l})$, and raised pro-brain natriuretic peptide (3648 ng/l). Other routine laboratory examinations, HIV tests, and autoantibody screening were normal. Right heart catheterisation revealed raised pulmonary pressure and resistance with reduced cardiac index (table 1), making a significant arteriovenous shunt highly improbable. ${ }^{7}$ Unfortunately, a shunt study while breathing 100\% oxygen was not performed initially and the patient later refused to be examined again. The PAP did not react to inhalation of $40 \mathrm{ppm}$ nitric oxide or to $10 \mu \mathrm{g}$ aerosolised iloprost, but pulmonary arterial resistance decreased by $10 \%$ with nitric oxide and by $21 \%$ with iloprost. The alveolo-arterial oxygen tension was $27 \mathrm{~mm} \mathrm{Hg}$, slightly above the upper normal limit (20 mm Hg). Lung function tests revealed normal respiratory volumes (forced expiratory volume in 1 second $\left(\mathrm{FEV}_{1}\right) 3.1 \mathrm{l}$, forced vital capacity (FVC) 4.56 l, total lung capacity 5.99 l, residual volume $1.43 \mathrm{l}$ ) with a slightly obstructive flow pattern $\left(\mathrm{FEV}_{\mathrm{l}} / \mathrm{FVC} 68 \%\right)$, and the carbon monoxide transfer factor was normal $(9.8 \mathrm{mmol} / \mathrm{kPa} / \mathrm{min})$. Oral anticoagulation with phenprocoumon and treatment with inhaled iloprost at a total daily dose of $50 \mu \mathrm{g}$ were started.

As the ventilation-perfusion lung scan showed small subsegmental perfusion defects and there is a known association between CTEPH and KTS, ${ }^{56}$ helical computed tomographic angiography and conventional angiography were performed. Unexpectedly, neither examination revealed signs of CTEPH. However, numerous small vascular malformations with multiple ectasias of peripheral pulmonary arterial vessel and vascular convolutes were seen in the left thoracoabdominal wall (fig 1). Surgical lung biopsy was offered but the patient declined.

Treatment with oral anticoagulation and inhaled iloprost was continued and was well tolerated. After 1 month of treatment the 6 minute walk distance improved by about $10 \%$ from $523 \mathrm{~m}$ to $574 \mathrm{~m}$ and the patient felt markedly better.

\section{DISCUSSION}

KTS is a rare syndrome (about 1500 recorded cases) of unknown cause. ${ }^{2}$ The main pulmonary complication of KTS is pulmonary thromboembolism with a frequency of $8-22 \%$ reported in larger series. ${ }^{12}$ Although the exact mechanism of

\footnotetext{
Abbreviations: $\mathrm{CTEPH}$, chronic thromboembolic pulmonary hypertension; HHT, hereditary haemorrhagic telangiectasia; KTS, Klippel-Trenaunay syndrome; PAH, pulmonary arterial hypertension; $P A P$, pulmonary arterial pressure; $\mathrm{PCH}$, pulmonary capillary haemangiomatosis; $\mathrm{PH}$, pulmonary hypertension; PVOD, pulmonary veno-occlusive disease
} 
Table 1 Haemodynamic and blood gas assessments

\begin{tabular}{|c|c|c|c|}
\hline & Baseline & $\begin{array}{l}\text { After inhalation } \\
\text { of NO } 40 \mathrm{ppm} \\
\text { for } 10 \mathrm{~min}\end{array}$ & $\begin{array}{l}30 \text { min after } \\
\text { inhalation of } \\
\text { iloprost } 10 \mu \mathrm{g}\end{array}$ \\
\hline Heart rate (beats/min) & 84 & 88 & 87 \\
\hline Mean arterial blood pressure $(\mathrm{mm} \mathrm{Hg})$ & 94 & 87 & 90 \\
\hline $\begin{array}{l}\text { Mean pulmonary arterial pressure } \\
(\mathrm{mm} \mathrm{Hg})\end{array}$ & 41 & 40 & 40 \\
\hline $\begin{array}{l}\text { Pulmonary arterial occlusion pressure } \\
(\mathrm{mm} \mathrm{Hg})\end{array}$ & 8 & 8 & 5 \\
\hline Right atrial pressure $(\mathrm{mm} \mathrm{Hg})$ & 4 & 2 & 0 \\
\hline Cardiac index $\left(\mathrm{l} / \mathrm{min} \cdot \mathrm{m}^{2}\right)$ & 1.7 & 1.9 & 2.1 \\
\hline $\begin{array}{l}\text { Pulmonary vascular resistance } \\
\left(\text { dyne. } / \mathrm{cm}^{5}\right)\end{array}$ & 851 & 711 & 717 \\
\hline Arterial oxygen saturation (\%) & 96.5 & 97.7 & 94.3 \\
\hline Arterial oxygen tension $(\mathrm{mm} \mathrm{Hg})$ & 75.8 & 83.4 & 67.8 \\
\hline Arterial carbon dioxide tension $(\mathrm{mm} \mathrm{Hg})$ & 31.3 & 25.3 & 29.6 \\
\hline Mixed venous oxygen saturation (\%) & 66.3 & 65.1 & 67.4 \\
\hline Alveolo-arterial oxygen tension $(\mathrm{mm} \mathrm{Hg})$ & 27 & 27 & 36 \\
\hline
\end{tabular}

the propensity to thromboembolism is not known, it seems reasonable to suggest that changes in venous flow make an important contribution. Signs of inherited thrombophilia have not been found in KTS patients with thrombosis. ${ }^{56}$
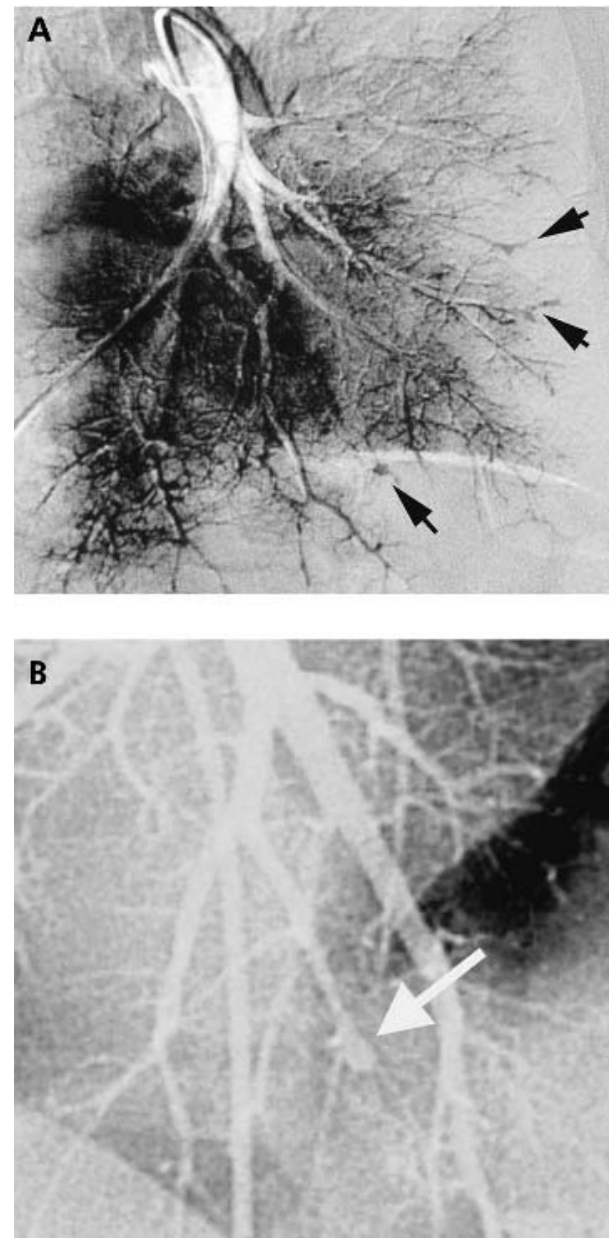

Figure 1 (A) Parenchymal phase of left lower lobe pulmonary digital subtraction angiography. Note ectasias of several terminal arterial branches (arrows). (B) Magnified unsubtracted angiogram of aneurysmatic terminal pulmonary artery (arrow).
Recurrent or unresolved pulmonary embolism may lead to CTEPH, and several patients with KTS and CTEPH have been reported. ${ }^{5}{ }^{6}$ Two underwent pulmonary thrombendarterectomy with a successful long term outcome. ${ }^{6}$ Although the lung scan in our patient showed some minor ventilation-perfusion mismatches, CTEPH was excluded by pulmonary angiography. However, multiple small peripheral pulmonary artery malformations were evident on helical CT scanning and conventional angiography which we interpreted as possibly being related to KTS.

Pulmonary small vascular malformations are thought to play an important pathogenetic role in other pulmonary vascular disorder such as veno-occlusive disease (PVOD) and pulmonary capillary haemangiomatosis $(\mathrm{PCH})$. In contrast to hereditary haemorrhagic telangiectasia (HHT, Osler's disease), ${ }^{7}$ another small vessel disease possibly leading to pulmonary hypertension, PVOD and PCH do not present with significant arteriovenous shunts as culprit lesions for $\mathrm{PH}$. The aetiology and pathogenesis of these rare disorders are not known; it is hypothesised that in PVOD a thrombotic diathesis may be responsible while, in PCH, a neoplastic proliferation and in HHT endothelial dysregulation due to mutation of the endoglin and activin receptor-like kinase 1 genes are thought to be involved. Despite the different morphological and anatomical involvement, diseases of this small pulmonary vessel may present with severe pulmonary hypertension which usually progresses rapidly to right cardiac failure without treatment. Although the common pathogenetic mechanisms of PAH are not known, pulmonary vascular changes of distinctive causes lead to endothelial dysfunction and activation of a cascade of different regulatory systems (coagulatory, inflammatory, proliferative) resulting in an imbalance in vasoconstriction and smooth muscle cell proliferation, the main culprit of $\mathrm{PH}^{8}{ }^{8}$ In KTS various manifestations of vascular malformations have been described $^{56}$ analogous to other pulmonary small vessel alterations (PVOD/PCH) which probably lead to similar alterations in vascular function and consecutive raised PAP, some of them might be associated with mutation in the gene encoding for the angiogenetic protein VG5Q, as recently described. ${ }^{9}$ In accordance with the clinical and radiomorphological findings in our patient, we attributed the pulmonary vascular malformation to KTS and hypothesise that they may be markers of a small vessel process leading to raised PAP.

Since other reasons for his $\mathrm{PH}$ - such as CTEPH or high cardiac output due to arteriovenous malformations (as seen in Parkes-Weber syndrome) and other diseases-were excluded, he was diagnosed as having PAH possibly related 
to small pulmonary vascular malformation in KTS. To date, published reports of PH in patients with KTS have attributed the PH to large vessel disease (that is, CTEPH). However, the work up of patients with PH and KTS may need to include small vessel disease. It is important to distinguish between the two forms because the treatment options are significantly different. ${ }^{10}$

\section{Authors' affiliations \\ S Ulrich, M Fischler, R Speich, Departement of Internal Medicine, \\ University Hospital of Zurich, Switzerland \\ B Walder, Hirslanden Clinic, Zurich, Switzerland \\ T Pfammatter, Department of Radiology, University Hospital of Zurich, Switzerland \\ Competing interests: none declared}

Correspondence to: Dr S Ulrich, Departement of Internal Medicine, University Hospital of Zurich, Switzerland; silvia.ulrich@usz.ch

Received 12 February 2004

Accepted 29 May 2004

\section{REFERENCES}

1 Baskerville PA, Ackroyd JS, Lea TM, et al. The Klippel-Trenaunay syndrome: clinical, radiological and haemodynamic features and management. $\mathrm{Br}$ J Surg 1985;72:232-6.

2 Jacob AG, Driscoll DJ, Shaughnessy WJ, et al. Klippel-Trenaunay syndrome: spectrum and management. Mayo Clin Proc 1998;73:28-36.

3 Parkes-Weber F. Haemangiectatic hypertrophy of limbs: congenital phlebarteriectasis and so-called congenital varicose veins. Br J Child Dis 1918;15:13-7.

4 Cohen, MM. Klippel-Trenaunay syndrome: editorial comment. Am J Med Genet 2000;93:171-5.

5 Muluk SC, Ginns LC, Semigran MJ, et al. Klippel-Trenaunay syndrome with multiple pulmonary emboli: an unusual cause of progressive pulmonary dysfunction. J Vasc Surg 1995;21:686-90.

6 Walder B, Kapelanski DP, Auger WR, et al. Successful pulmonary thromboendarterectomy in a patient with Klippel-Trenaunay syndrome. Chest 2000;117:1520-2.

7 Gossage JR, Kanj G. Pulmonary arteriovenous malformations. A state of the art review. Am J Respir Crit Care Med 1998;158:643-61.

8 Eddahibi S, Morrell N, d'Ortho MP, et al. Pathobiology of pulmonary arterial hypertension. Eur Respir J 2002;20:1559-72.

9 Tian XL, Kadaba R, You SA, et al. Identification of an angiogenic factor that when mutated causes susceptibility to Klippel-Trenaunay syndrome. Nature 2004;427:640-5

10 Chatterjee K, De Marco T, Alpert JS. Pulmonary hypertension: hemodynamic diagnosis and management. Arch Intern Med 2002;162:1925-33.

\section{LUNG ALERT}

\section{Acid suppression and paediatric asthma}

A Stordal K, Johannesdottir GB, Bentsen BS, et al. Acid suppression does not change respiratory symptoms in children with asthma and gastro-oesophageal reflux. Arch Dis Child 2005;90:956-60

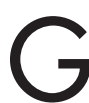
astro-oesophageal reflux is common in children with asthma but its relevance is unclear. The effects of proton pump inhibitors on asthma control in children are not known.

Thirty six children aged 7-16 years with mild to moderate asthma and symptomatic gastro-oesophageal reflux (identified on 24 hour oesophageal $\mathrm{pH}$ monitoring) were randomised to 3 months of treatment with omeprazole $20 \mathrm{mg}$ once daily or placebo. There were no significant differences in asthma symptoms, quality of life, lung function, or short acting bronchodilator use between the omeprazole and placebo groups. Repeat oesophageal pH studies in a subset of children receiving omeprazole indicated adequate acid suppression. The results of this study argue against a causal relationship between acid gastro-oesophageal reflux and asthma morbidity, and are consistent with the findings of a recent Cochrane database meta-analysis.

The authors conclude that proton pump inhibitors are unhelpful in children with asthma who are already receiving conventional asthma treatment.

S Birring Specialist Registrar in Respiratory Medicine, Glenfield Hospital, Leicester, UK; sb134@le.ac.uk 